\title{
Brandenburgische Erwägungen für eine Stärkung der Autonomie der Dritten Gewalt
}

Der Minister der Justiz des Landes Brandenburg hat im Frühjahr 2010 die Projektgruppe „Richterliche Selbstverwaltung“" ins Leben gerufen.*

Der Gruppe wurde der Auftrag erteilt, in einem ersten Schritt Vorschläge zu dem Entwurf eines gemeinsamen Richtergesetzes für die Länder Berlin und Brandenburg zu erarbeiten (A.). In einem zweiten Schritt hat die Projektgruppe grundsätzliche Überlegungen zu einer unabhängig sich verwaltenden, autonomen Justiz angestellt (B.). Welche konkreten Folgerungen sich daraus ergeben, ist in einem weiteren Schritt zu erarbeiten. Vor allem muss auch die Stellung der Staatsanwaltschaft in einem System umfassender Autonomie nach einer wissenschaftlich und rechtspolitisch angeleiteten Bestandsaufnahme neu definiert werden. ${ }^{1}$ Die Positionen anderer Dienstgruppen innerhalb des Justizsystems sind ebenfalls zu überdenken.

\section{A. Erster Schritt: Sachverständige Beratung zum gemeinsamen Richtergesetz der} Länder Berlin und Brandenburg (1. Arbeitspapier)

Der bis Ende Juni 2010 von der Projektgruppe beratene und vom Ministerium der Justiz veröffentlichte Entwurf ${ }^{2}$ war Gegenstand der Verhandlungen des Brandenburgischen Justizministeriums mit der Berliner Senatsverwaltung für Justiz über den Entwurf des Gesetzes zur Angleichung des Richterrechts in den Ländern Berlin und Brandenburg. Auch wenn sich die Vorschläge der Projektgruppe in entscheidenden Passagen jetzt nicht in dem mit Berlin abgestimmten aktuellen Gesetzesentwurf niedergeschlagen haben, so kann man sie doch als einen zukunftsgerichteten Musterentwurf eines Landesrichtergesetzes ansehen.

* Dieser Projektgruppe gehören an:

Prof. Dr. Peter-Alexis Albrecht, Goethe-Universität, Frankfurt am Main

Präsident des Landgerichts i. R. Hans-Ernst Böttcher

Präsident des Landgerichts Cottbus Klaus-Christoph Clavée

Ministerialrat Dr. Georg Kirschniok-Schmidt

Präsident des Verwaltungsgerichts Cottbus Andreas Knuth

Direktor des Amtsgerichts i. R. Hans-Joachim Pauckstadt

Vorsitzende Richterin am Oberlandesgericht Ramona Pisal

Generalstaatsanwalt Dr. Erardo Cristoforo Rautenberg

Vorsitzender Richter am Finanzgericht i. R. Heinz Stötzel.

1 Vgl. die Beiträge zur Unabhängigkeit der Staatsanwaltschaft in KritV 3/2010.

2 www.mdj.brandenburg.de/cms/detail.php/bb1.c.251096.de. 


\section{Der Entwurf im Überblick}

Bereits in dem Entwurf hat die Projektgruppe darauf geachtet, ihn mit weitergehenden Überlegungen zur Fortschreibung in Richtung einer autonomen Justiz kompatibel zu halten, wie sich aus dem ersten Arbeitsbericht ergibt:

,(...) 2. Der vorgelegte überarbeitete Gesetzesentwurf

- hält bewährte Elemente des brandenburgischen und des Berliner Richtergesetzes aufrecht und schreibt sie fort;

- trägt verstärkt der Gleichstellung Rechnung;

- nimmt modernere Regelungen aus anderen Ländern der Bundesrepublik Deutschland auf;

- berücksichtigt die Kompetenzverschiebungen zwischen Bund und Ländern nach der Föderalismusreform II.

3. Daneben hat die Projektgruppe darauf geachtet, im Bereich des Richterrechts zukünftig weitere Elemente zu verankern, die die Unabhängigkeit der Richterinnen und Richter (und vermehrt auch der Staatsanwältinnen und Staatsanwälte) sichern, ähnlich wie es der Bundesgesetzgeber 1999 mit dem Gesetz zur Stärkung der Unabhängigkeit der Richter und Gerichte zur Reform der Präsidien der Gerichte (§ 21 a ff GVG) getan hat.

\section{Im Einzelnen:}

3.1. Traditionelle Elemente zur Stärkung der Unabhängigkeit der Richterinnen und Richter sind

a) die (soeben schon erwähnten) Präsidien der Gerichte, die zugleich für die Garantie des gesetzlichen Richters (Art. 101 Abs. 1 Satz 2 GG) bedeutsam sind. Im Präsidium verwalten die Richterinnen und Richter ihr Gericht selbst, allerdings beschränkt auf die Bereiche Zusammensetzung der Spruchkörper und Zuweisung der richterlichen Aufgaben;

b) die Mitbestimmung gegenüber der Justiz- und Gerichtsverwaltung durch Richterräte und Präsidialräte;

c) die Richterdienstgerichte, die gewährleisten, dass gravierendere Disziplinarmaßnahmen als der Verweis von unabhängigen Richterinnen und Richtern und nicht von einem Organ der Verwaltung ausgesprochen werden. Überdies gibt $§ 26$ III DRiG der einzelnen Richterin und dem einzelnen Richter, die oder der sich in ihrer/seiner Unabhängigkeit beeinträchtigt sieht, das Recht, selbst das Richterdienstgericht anzurufen;

d) die Richterwahlausschüsse (in den Ländern, soweit eingerichtet).

\subsection{Vorstellungen für die Zukunft}

a) In Zusammenhang mit dem zukünftigen Richtergesetz für Berlin und Brandenburg hat sich die Projektgruppe zunächst nicht mit dem Recht der Präsidien der Gerichte befasst. Auf der ,zweiten Stufe“ wird zu fragen sein, ob und wie die Selbstverwaltungsbefugnisse des Präsidiums auszubauen sind und auf allen Ebenen der Gerichts- 
und Justizverwaltung bis hin zur Länderebene Selbstverwaltungsorgane vorzusehen sind.

b) Die Mitbestimmung wird hinsichtlich der Regelungsgegenstände („Allzuständigkeit") und hinsichtlich des Mitbestimmungsgrades (durchgängig echte Mitbestimmung, nirgendwo bloße Mitwirkung) ausgebaut. Die Projektgruppe empfiehlt mehrheitlich ferner, die bisherigen Kompetenzen des Präsidialrats auf den Gesamtrichterrat zu übertragen, soweit dies nach (reformiertem) Bundesverfassungsrecht zulässig ist.

c) Die Disziplinarbefugnisse sieht die Projektgruppe bei den Richterdienstgerichten in guten Händen. Diese sollten (als echte Gerichte) aber nach wie vor nur mit Richterinnen und Richtern besetzt sein, also nicht (wie von Berlin vorgeschlagen) auch ein anwaltliches Mitglied haben.

d) In der Zusammensetzung des Richterwahlausschusses wird die richterliche Komponente gestärkt. Weiter ist die Projektgruppe der Auffassung, dass auf die Quote der parlamentarischen Mitglieder des Ausschusses auch solche Persönlichkeiten wählbar sein sollten, die nicht selbst dem Parlament angehören (s. schon jetzige Regelungen u.a. in Berlin und Hamburg). Schließlich wird insgesamt das Gewicht des Ausschusses gegenüber dem Minister/der Ministerin gestärkt.

3.3. Die richterliche Unabhängigkeit will die Projektgruppe mehrheitlich auch dadurch gestärkt sehen, dass - außer bei den Richterinnen und Richtern auf Probe - Regelbeurteilungen entfallen. Auf Lebenszeit ernannte Richterinnen und Richter sollen nur auf Antrag beurteilt werden (u.a. aus Anlass einer Bewerbung).

4. Insgesamt war es das Bestreben der Projektgruppe, die vorgeschlagenen Regelungen für das zukünftige gemeinsame Richterdienstrecht in Berlin und Brandenburg so zu gestalten, dass sie den weiteren Weg in Richtung einer Selbstverwaltung/Autonomie der Justiz fördern und schon jetzt absehbar mit zukünftigen weitergehenden Regelungen verträglich sind.

Sie hat sich dabei von dem Gedanken leiten lassen, dass zukünftig erweiterte Selbstverwaltungsstrukturen nicht nur für die Gerichte und damit für die Richterinnen und Richter, sondern auch für die Staatsanwaltschaft und damit für die Staatsanwältinnen und Staatsanwälte zu schaffen sind."

Hinweise zu Schwerpunkten und Einzelregelungen finden sich auf der Homepage des Ministeriums der Justiz des Landes Brandenburg. ${ }^{3}$

\section{B. Zweiter Schritt: Erwägungen für eine Stärkung der Autonomie der Dritten Gewalt (2. Arbeitspapier)}

Im Juni 2011 legte die Projektgruppe ihr zweites Arbeitsergebnis vor.

Die Projektgruppe ist in ihrer Gesamtheit zu dem Ergebnis gekommen, mehr Autonomie für die Dritte Gewalt zu fordern. Sie hat das Pro und Contra einer vollständigen Abkopplung der Verwaltung der Justiz von der Exekutive erörtert. Im Folgenden werden den befürwortenden Argumenten aus der Projektgruppe die Contra-Argumente der ge-

3 www.mdj.brandenburg.de/cms/detail.php/bb1.c.251104.de. 
genteiligen Auffassung in kontrastierender Intention vorab jeweils kursiv vorangestellt. Nach im Einzelnen durchaus kontroverser Diskussion und Bewertung hat die Projektgruppe mit großer Mehrheit das nachfolgende Positionspapier verabschiedet.

\section{ERSTENS}

\section{Gewaltenteilung: Gewaltenverschränkung versus Gewaltenkontrolle? \\ - Verwirklichung des Allgemeinwohls oder Freiheitsschutz vor öffentlichem Machtmissbrauch -}

\section{CONTRA}

Gewaltenverschränkung: Justiz ist Aufgabe aller Gewalten im Sinne einer Gewaltenbalance

- Das deutsche System der Gewaltenteilung ist eines der Gewaltenbalance

Das deutsche System der Gewaltenteilung ist nicht auf völlige Trennung der Gewalten gerichtet, sondern auf eine Trennung der Verantwortungsbereiche staatlicher Gewalt (Gewaltengliederung) ungeachtet wechselseitiger Bezüge, Verschränkungen und Abhängigkeiten. Es ist ein System der Gewaltenbalance, gerichtet auf die Kontrolle und Begrenzung staatlicher Macht, aber auch zugleich gerichtet auf die organadäquate und funktionsgerechte Verteilung der Staatsaufgaben zur Verwirklichung des Gemeinwohls und zur Sicherung der individuellen Grundrechte. Gewaltenteilung ist auf eine rationale und sachgerechte Organisation des Staates gerichtet, die gewährleistet, dass staatliche Entscheidungen möglichst von den Organen getroffen werden, die dafür die besten Voraussetzungen erfüllen.

- Die Exekutive hat nach Maßgabe einer Fülle von Gesetzen für die Dritte Gewalt verwaltungsmäßige Dienstleistungen zu erbringen

Die Justiz - als Inbegriff aller Einrichtungen und Vorkehrungen, die der Erfüllung des Justizgewährungsanspruchs und des Rechtsschutzauftrags dienen - ist in Deutschland eine Aufgabe aller drei Staatsgewalten: Die rechtsprechende Gewalt selbst ist allein ,, den Richtern anvertraut" und wird durch die Gerichte ausgeübt (Art. 92 GG). Ausschließlich ihnen obliegt die eigentliche Rechtsprechung, insbesondere die Aufgabe, in einem fairen und zügigen Verfahren dem Bürger Rechtsschutz zu gewähren. Die Gesetzgebung hat durch gesetzliche Regelungen zur Gerichtsverfassung, zum Gerichtsverfahren, zur Gerichtsorganisation, zur Rechtsstellung der Richterinnen und Richter einschließlich ihrer Berufung, Besoldung und Versorgung sowie durch die Gewährung von Haushaltsmitteln die Grundlage dafür zu schaffen, dass die Gerichte ihre Aufgabe wahrnehmen können. Die Exekutive hat die Aufgabe, unter Wahrung der richterlichen Unabhängigkeit im Rahmen und nach Vorgabe der Gesetze die verwaltungsmäßigen Dienstleistungen zu erbringen, damit die funktionsgemäße Organisation der Gerichte, 
die Verteilung der personellen und sachlichen Mittel in einer Weise gewährleistet werden, die ihnen die wirksame Verwirklichung des Rechtsschutzauftrags ermöglichen.

- Die Sorge um Gewährleistung funktionierender Rechtsprechung ist Aufgabe der Exekutive

Zu den Exekutivaufgaben gehört die ständige Sorge um die Gewährleistung des Rechtsprechungsauftrags entsprechend den Erwartungen der Rechtsschutzsuchenden und dem Verfassungsauftrag (Rechtsschutz in angemessener Zeit). Hierzu gehören die laufende Erfassung des finanziellen Bedarfs der Gerichte, die Prüfung der notwendigen Planstellen, schließlich aber auch die laufende Überprüfung, welche (u.U. gesetzlichen) Änderungen erforderlich sind, um Überforderungen des Rechtssystems und Überlastungen der Gerichte zu vermeiden (Verfahrensrecht, materielles Recht). Die Vorbereitung gesetzgeberischer Entscheidungen und die Beratungsleistungen gegenüber dem zuständigen (Bundes- oder Landes-)Gesetzgeber ist eine der wichtigsten Aufgaben (auch) der Justiz- und Rechtspolitik.

- Die Unabhängigkeit der Rechtsprechung bedingt keine Eigenständigkeit in der Verwaltung der Judikative

In die Exekutivaufgaben sind die Gerichte notwendigerweise selbst einbezogen. Aus der Eigenständigkeit ihrer Rechtsprechungsfunktion im Rahmen der Gewaltenteilung folgt jedoch nicht, dass sämtliche Exekutivaufgaben ihnen überlassen werden müssten, weil - wie von den Vertretern der Selbstverwaltungsidee angeführt - sonst die Unabhängigkeit ihrer Rechtsprechung gefährdet wäre. Exekutivaufgaben liegen in der Gesamtverantwortung der hierfür legitimierten und parlamentarisch kontrollierten Exekutive.

PROjektgruppe

Gewaltenkontrolle: Justiz ist ausschließlich Sache der Dritten Gewalt

- Gewaltenkontrolle ist kein historischer oder gar überholter Begriff des Verfassungsrechts. Sie dient in der Demokratie der Erkenntnis, dass eine effektive Kontrolle der Staatsgewalten das zentrale Element des Rechtsstaats ist. Diese Kontrolle verwirklicht den Freiheitsschutz der Bürgerinnen und Bürger durch Recht.

\section{- Gewaltenkontrolle effektivste Funktionsgarantie der Freiheit}

Staatliche Gewalt darf nicht ungeteilt in einer einzigen Hand liegen - das lehrt das Gewaltenteilungsprinzip. Im Vordergrund steht dabei die Intention, den Missbrauch staatlicher Gewalt abzuwehren. In einem parlamentarischen Regierungssystem wird zwar eine lückenlose Trennung staatlicher Zuständigkeiten und Befugnisse nicht aus- 
nahmslos verwirklicht und es gibt stattdessen Bereiche, in denen die Staatsgewalt mehreren Organen gewissermaßen zur gesamten Hand übertragen wird (sog. Gewaltenverschränkung). Man spricht dann davon, dass im Prinzip der Gewaltenteilung der Systembruch quasi systemimmanent angelegt ist. Wenn hierfür auf den Aspekt der Gewaltenbalance verwiesen wird, ist ungeachtet dessen die Idee der gegenseitigen Kontrolle der Staatsgewalten nicht zu vergessen. Ohne sie wird der Grundsatz ad absurdum geführt. Wenn ein Organ von einem anderen in seinen Entscheidungen und Verhaltensweisen abhängig ist (z.B. durch Einfluss auf die personelle Besetzung), so liegt zwar eine funktionelle Verteilung, mit Sicherheit aber keine Gewaltenteilung vor. Gewaltenkontrolle ist daher trotz zahlreicher Elemente von Gewaltenverschränkung die effektivste Funktionsgarantie der Freiheitssicherung und der Mehrung des Gemeinwohls im demokratischen Verfassungsstaat.

\section{- Gewaltenteilung impliziert Spannungsverhältnis, nicht Harmonie}

Dass die Justiz eine Aufgabe aller Staatsgewalten wäre, mag in Deutschland traditionell so gewachsen sein, erscheint jedoch historisch überholt. Die Loslösung der Justiz aus dem Zugriff der Exekutive widerspräche hingegen nicht dem Gewaltenteilungsprinzip, sondern hilft seiner Verwirklichung. Gerade im Hinblick auf die seit der Schaffung des Grundgesetzes stark veränderten Bedingungen wäre eine autonome Dritte Gewalt wünschenswert und würde dem Montesquieu'schen Grundgedanken der Gewaltenkonkurrenz besser gerecht: Dem Prinzip innewohnend ist der Umstand, dass die Personen bzw. die Personengruppen, denen die einzelnen Staatsgewalten anvertraut sind, zueinander in einem Spannungsverhältnis stehen.

\section{- Parteiendemokratie führt zur Gewaltenhäufung und schwächt den Kontrollansatz}

Der Übergang zu einer Parteiendemokratie hat das Spannungsverhältnis zwischen den Trägern der Legislative und der Exekutive größtenteils beseitigt. Durch das Konglomerat von Parlamentsmehrheit und Regierung wird die Gewaltenteilung von einer Gewaltenhäufung abgelöst. Die Trennung der beiden Staatsgewalten ist kein wirksames politisches Machtverteilungsprinzip mehr, sondern sinkt zu einem Prinzip der innerstaatlichen Zuständigkeitsverteilung herab.

Vor diesem Hintergrund wird - so Roman Herzog - die Dritte Gewalt zum eigentlichen Garanten der Gewaltenteilungsidee, zum entscheidenden Faktor der effektiven Kontrolle staatlicher Macht. Durch den in Art. 19 IV GG garantierten Rechtschutzanspruch gegenüber öffentliche Handlungen und die Möglichkeit der abstrakten Normenkontrolle wird jeder Hoheitsakt von Legislative und Exekutive überprüfbar. Insbesondere (aber nicht nur) die Verwaltungsgerichtsbarkeit gewinnt somit bei der Bildung eines ausgleichenden Machtzentrums an Bedeutung. Das hat schon Wilhelm von Humboldt 1792 in seiner Schrift „Ideen zu einem Versuch die Grenzen der Wirksamkeit des Staates zu bestimmen“" erkannt. 
- Unvereinbarkeit von exekutiver Prägekraft und Kontrollauftrag der Dritten Gewalt

Effektive Gewaltenkontrolle kann jedoch nicht stattfinden, wenn die zu kontrollierende Exekutive ihre Kontrolleure selbst auswählt, bestellt, bezahlt und befördert. Diese das Grundprinzip von Gewaltenkontrolle aushebelnden Organisationsmechanismen sind in fast allen Mitgliedsstaaten der EU verfassungsrechtlich eliminiert und dürfen auch aus der Sicht des Europarates das Leitbild unabhängiger Rechtsprechung nicht in Frage stellen. Zwar ist die richterliche Unabhängigkeit im Sinne spruchrichterlicher Tätigkeit gemäß Art. 97 GG als Justizgrundrecht gewährt und findet nachdrückliche Beachtung. Es erscheint aber andererseits eine indirekte Beeinflussung der Unabhängigkeit seitens der Exekutive möglich, so durch die Gestaltung der Arbeitsbedingungen, insbesondere Ressourcenzuweisung, und durch Personalentscheidungen.

\section{- Rechtspolitische Konsequenzen aus Sicht der Berufsverbände der Dritten Gewalt}

Alle in der Bundesrepublik Deutschland bestehenden allgemeinen richterlichen Berufsorganisationen sprechen sich inzwischen für eine Veränderung der Justizverwaltung in Richtung Autonomie aus. Nach Auffassung des Deutschen Richterbundes (DRB), der Neuen Richtervereinigung (NRV) und der Richterinnen und Richter in ver.di muss die Organisation der Justiz von der Exekutive vollständig abgekoppelt werden. Gewaltenkontrolle als Freiheitsschutz und als Schutz vor öffentlichem Machtmissbrauch erfordert eine verfassungsrechtlich und institutionell gesicherte Selbstverwaltung der Justiz. So kann es gelingen, die „Einbuße an Rechtsstaatlichkeit, die die Einebnung des zwischen Legislative und Exekutive ursprünglich bestehenden Spannungsverhältnisses durch die politischen Parteien mit sich gebracht hat" (Herzog in: Maunz/Dürig, GG, Art. 20, Rn. 36), auszugleichen.

\section{ZWEITENS}

Einbuße an demokratischer Legitimation durch Selbstverwaltung versus Demokratiestärkung durch unmittelbare Mitwirkung der Parlamente bei der Richterwahl

CONTRA

Selbstverwaltung verhindert Rückkopplung der Justizverwaltung an demokratische Kontrolle

\section{Parlamentarische Kontrolle verlangt Regierungsverantwortung}

- Letztverantwortung der Regierung für alle exekutiven Aufgaben

Aus dem System der Gewaltenteilung folgt, dass grundsätzlich alle exekutiven Aufgaben in der Letztverantwortung der Regierung wahrgenommen werden (keine ,,mi- 
nisterialfreien" Räume). Die Regierung nimmt ihre Verantwortung gegenüber dem Parlament wahr, dessen Aufgabe die Kontrolle der Regierung, aber auch die Teilnahme an der Staatsleitung ist. Das Parlament nimmt als Repräsentant des Volkes seine Funktion nicht allein durch die Akte der Gesetzgebung wahr, sondern dadurch, dass es den laufenden gesellschaftlichen Willensbildungsprozess aufgreift, parlamentarisch verarbeitet und der Regierung durch Kritik und Anregung Impulse zur Umsetzung politischer und gesetzlicher Entscheidungen gibt. Die parlamentarischen Kontrollrechte sind gegen die Regierung gerichtet und mittelbar auf den gesamten der Regierungsverantwortung unterstehenden Verwaltungsapparat. Initiativen zur Gesetzgebung sind das Ergebnis eines ständigen kommunikativen Austauschs, der in Achtung der jeweiligen verfassungsrechtlichen Funktionen zwischen Parlament und Regierung stattfindet. Dies gilt auch für die Frage, mit welchen normativen Bestimmungen die Justizgewährung im Land gesichert werden soll.

- Verselbständigung der Justizverwaltung ist Beschneidung des Kompetenzbereichs des Parlaments

Wo es an Regierungsverantwortung fehlt, fehlt dem Parlament die Möglichkeit zur Kontrolle, zur Information, zur eigenen Willensbildung und zur gesetzgeberischen Einflussnahme. Exekutive Bereiche, die außerhalb der Regierungsverantwortung liegen, sind Ausnahmefälle, für die eine besondere verfassungsrechtliche Rechtfertigung und besondere Mechanismen zur Wahrung der parlamentarischen Rechte vorgesehen sind (Rechnungshof, Verfassungsgericht, Datenschutzaufsicht). Für den Gesamtbereich der Justiz ist eine Kompetenzbeschränkung des Parlaments, die in der Herauslösung sämtlicher Justizangelegenheiten aus dem Verantwortungsbereich der Regierung läge, nicht zu verantworten.

\section{Selbstverwaltung führt zu Einbuße an demokratischer Legitimation}

- Justizverwaltung ist nicht von der richterlichen Unabhängigkeitsgarantie erfasst

Justizverwaltung bleibt Exekutive unabhängig davon, ob sie der Regierungsverantwortung untersteht oder ob sie von besonderen (Selbstverwaltungs-) Organen wahrgenommen wird. Justizverwaltung bedarf daher - bis auf bestimmte Formen von gerichtlicher Selbstverwaltung, die sich aus der Unabhängigkeit der Rechtsprechung ergeben (z. B. Geschäftsverteilung) - notwendigerweise der Rückkopplung an demokratische Verfahren, aus denen sie ihre Legitimation erlangt.

- Unkontrollierte Justizverwaltung wird illegitimes Machtzentrum

Die Forderung nach einer Selbstverwaltung oder nach einer Autonomie der Justiz gipfelt in der Forderung nach einer völligen Loslösung der Justizverwaltung aus dem Verantwortungsbereich der Regierung. Justiz verwaltet sich selbst, heißt: Sie ist nie- 
mandem gegenüber verantwortlich als sich selbst. Es mögen zwar Modelle realisiert werden, wonach an der Spitze der autonom wirkenden Justizverwaltungsorgane Personen stehen, die in einem noch näher zu bestimmenden Verfahren demokratisch legitimiert sind, weil sie über parlamentarisch gebilligte Mehrheitsentscheidungen in ihre Funktionen geraten. Dies täuscht jedoch nicht darüber hinweg, dass es an einer laufenden Rückkopplung exekutiver Aufgabenwahrnehmung an das Parlament fehlt. Gerade diese Rückkopplung ist jedoch entscheidend, um ein „Machtzentrum “ außerhalb parlamentarischer Kontroll- und Einflussmöglichkeiten zu verhindern.

- Nur Rechtsprechungsaufgaben gebührt das verfassungsrechtliche Unabhängigkeitsprivileg

Der Rechtsprechung ist es wesenseigen, dass sie keiner demokratischen Verantwortlichkeit und keinen Weisungen unterliegt. Richterliche Entscheidungen sind legitimiert durch ihre Bindung an das Gesetz und durch die persönliche Legitimation derjenigen, die zu Richterinnen und Richtern ernannt worden sind. Die Ernennung (Auswahl, Beförderung) muss auf das Volk rückführbar sein und verleiht den Richterinnen und Richtern demokratische Legitimation zur Ausübung der Staatsgewalt. Sind Wahlausschüsse oder sonstige Gremien für die Personalentscheidung zuständig, so müssen diese ihrerseits demokratisch legitimiert sein, damit die Legitimationskette nicht unterbrochen wird. Welche Folgerungen hieraus für die Zusammensetzung und Beschlussfassung der Richterwahlausschüsse zu ziehen sind, ist im Einzelnen umstritten. Hierfür gibt es unterschiedliche Modelle. Unzweifelhaft können Wahlgremien, die durch parlamentarische Mitwirkung hinreichend legitimiert sind, den Gewählten die Legitimation zur Ausübung ihrer Rechtsprechungsaufgabe vermitteln.

- Justizielle Verwaltungstätigkeit erfordert dauerhafte parlamentarische Kontrolle mittels des Prinzips der Regierungsverantwortung

Die Wahrnehmung von Exekutivaufgaben (Personal-, Organisations-, Haushaltsaufgaben, Mitwirkung an rechtspolitischen Fragen etc.) ist keine Rechtsprechungsaufgabe. Sie bedarf auch in Form der Justizverwaltung derselben demokratischen Legitimation wie andere Formen exekutiver Tätigkeit. Diese wird nicht bereits durch die persönliche Legitimation der Richterinnen und Richter oder der nichtrichterlichen Bediensteten vermittelt, die sich im einmaligen Akt ihrer Ernennung (Einstellung) erschöpft, sondern bedarf der ständigen Rückkopplung an das Parlament als Repräsentant des Volkes. Die Ausübung von (exekutiver) Staatsgewalt darf nicht autonom werden, sondern muss sich dauerhaft auf den Volkswillen zurückführen lassen und gegenüber dem Volk verantwortet werden, dessen Wille im Parlament repräsentiert wird. Demokratische Kontrolle verlangt eine ständige Beziehung zwischen den Gewalten, die nach dem herkömmlichen System gerade durch den Justizminister als demokratisch legitimiertem und parlamentarisch verantwortlichem Amtsträger vermittelt wird. Die demokratische Legitimation der Justizverwaltung wäre beeinträchtigt, wenn sie letztverantwortlich in die Hände von unabhängigen Organen gelegt würde, die keiner - oder faktisch nur einer einge- 
schränkten - parlamentarischen Verantwortung unterliegen. Hierdurch würde der Legitimationszusammenhang zerrissen. Das Defizit an demokratischer Legitimation aufgrund mangelnder Rückkopplung der Justizverwaltung an das Parlament lässt sich schwerlich durch Mechanismen wie Berichtspflichten oder dergleichen ausgleichen.

PROjektgruppe

Selbstverwaltung stärkt die unmittelbare demokratische Legitimation

\section{Parlamentarische Kontrolle ist im Modell der Selbstverwaltung garantiert}

- Kein Omnipotenzanspruch der Exekutive für Verwaltungsaufgaben

Das Grundgesetz hat in Art. 92 GG die rechtsprechende Gewalt den Richtern anvertraut. Die Verwendung des Begriffs „Gewalt" und der Grundsatz der Gewaltenteilung sprechen dafür, dass mehr gemeint ist als die bloße Kerntätigkeit der Rechtsprechung, dass vielmehr auch die organisatorische Dimension mit umfasst wird. Hierarchisch gegliederte Justizbehörden, die von der Verwaltung der Landesregierungen personell und sachlich abhängig sind und entsprechend gesteuert werden, widersprechen diesem verfassungsrechtlichen Auftrag diametral. Die Annahme, dass ein Verlust an Regierungsverantwortung für die Verwaltung der Justiz mit einem Verlust an demokratischer Legitimation einhergeht, basiert auf einem Omnipotenzanspruch der Exekutive, der keine Stütze im Grundgesetz findet.

\section{- Demokratische Legitimation mittels direkter Kontrolle durch das Parlament}

Der Rückschluss, dass das Parlament keine Möglichkeit zur Kontrolle, zur eigenen Willensbildung oder zur gesetzgeberischen Einflussnahme habe, wo es an Regierungsverantwortung fehle, kann nicht pauschal gezogen werden. Das Modell einer selbstverwalteten Justiz wäre viel eher in der Lage, die demokratische Legitimation zu verstärken - und zwar auf mehreren Wegen: Einerseits durch eine angemessene unmittelbare Mitwirkung des Parlaments bei der Richterwahl, andererseits durch näher zu konkretisierende Berichts- und Rechenschaftspflichten eines demokratisch legitimierten Selbstverwaltungsorgans (z.B. Justizverwaltungsrat oder Gerichtsbarkeitsräte) gegenüber dem Parlament im Sinne einer kontinuierlichen demokratischen Kontrolle. Mitwirkungsrechte des Parlaments können durch Vertretung der Parlamentarier im Justizverwaltungsrat direkt oder durch von ihnen berufene Kompetenzträger gestärkt werden.

\section{- Größere Sachnähe für justizspezifische Verwaltung ohne exekutive Einflussnahme}

Die Unabhängigkeit der Dritten Gewalt gebietet geradezu eine Zuständigkeit der Justiz für die Justizverwaltung. Unzulässige exekutive Einflussnahmen würden ausgeschlossen. Die Grenzziehung zwischen Verwaltungs- und Rechtsprechungsaufgaben kann justizintern viel sachnäher vorgenommen werden. Parlamentarische Kontrolle 
kann durchaus auch unmittelbar an die Justizverwaltung gekoppelt werden und macht Regierungsverantwortung - über die Person des Justizministers - überflüssig.

\section{Demokratiestärkung durch unmittelbare Mitwirkung des Parlaments bei der Richterwahl}

\section{- Modell der direkten demokratischen Legitimation durch das Parlament}

Eine wesentliche Demokratiestärkung erfährt eine selbstverwaltete Justiz durch eine unmittelbare Mitwirkung des Parlaments bei der Richterwahl. Nach den Reformmodellen der Deutschen Richterverbände wird die Auswahl und Einstellung von Richtern gegenüber der jetzigen mittelbaren demokratischen Legitimation durch die jeweiligen Landesregierungen ersetzt durch ein Modell der direkten Legitimation durch das Parlament. Die bisherigen Richterwahlausschüsse im Bund (verbindlich, s. Art. 95 Abs. 2 GG) und in einigen Bundesländern (fakultativ, s. Art 98 Abs. 4 GG) - wenn auch leider nicht in allen - haben bereits wichtige demokratische Voraussetzungen in Richtung Selbstverwaltung geschaffen, an die eine autonome und selbstverwaltete, demokratisch unmittelbar legitimierte Justiz anknüpfen kann.

Nach den Vorschlägen der Richterverbände sollen in Zukunft die Abgeordneten der zuständigen Parlamente (oder von ihnen entsandte Dritte) entweder zur Hälfte (DRB), mit Mehrheit (ver.di) oder zu zwei Dritteln (NRV) Mitglieder der Justizwahl- bzw. Richterwahlausschüsse sein. Damit soll der Legislative zurecht ein entscheidender Einfluss auf die Auswahl und Einstellung von Richtern eingeräumt werden. Die unmittelbare demokratische Legitimation bei der Richterwahl durch die Legislative stärkt, wie heute schon im Bund und in den Ländern mit (echtem) Richterwahlausschuss, das Prinzip der unmittelbaren Demokratie durch eine Optimierung der Legitimationskette.

\section{- Kein Demokratiedefizit durch Selbstverwaltung}

Die traditionelle und in Deutschland vorherrschende Orientierung sieht die parlamentarische Verantwortung durch die jeweils zuständige Landesregierung (bzw. für die Bundesgerichte durch die Bundesregierung) als hinreichend an. Ein Fehlen dieser parlamentarischen Verantwortung wird als Demokratiedefizit angesehen. Dies ist allerdings nur eine mittelbare demokratische Legitimationskette, die über die Exekutive einschließlich ihrer gesonderten Prägekräfte vermittelt wird. Ganz im Gegenteil führt erst eine unmittelbare Mitwirkung der Parlamente bei der Richterwahl im Sinne einer Stärkung von Gewaltenkontrolle und Gewaltenbalance sowie andere Mechanismen direkter Kontrolle zu einer Verstärkung der unmittelbaren Legitimation der Dritten Gewalt und zugleich zu einer deutlichen Demokratiestärkung durch Einbeziehung der Legislative.

Neben der verstärkten unmittelbaren Legitimation der Dritten Gewalt ist die fortlaufende Rückkopplung an das Parlament zu garantieren, die auch in einer autonomen Justizverwaltung notwendig ist. Hier sind gesetzlich klar definierte Berichts- und Rechen- 
schaftspflichten einzurichten. Die Kommunikation mit dem Parlament wird durch gemeinsame Organe (z.B. Richterwahlausschuss/Justizverwaltungsräte bzw. Gerichtsbarkeitsräte) sogar institutionalisiert.

Zusammenfassend: Eine selbstverwaltete Justiz kann eine stärkere - weil unmittelbare und dauerhafte - demokratische Kontrolle gewährleisten. Sie ist der mittelbaren Kontrolle durch Regierungsverantwortung damit überlegen. Darüber hinaus bietet sie eine verstärkte demokratische Legitimation durch die Einbindung des Parlaments in die Richterwahl und durch die Ersetzung eines politisch-interessengebundenen Organs (Exekutive) durch ein unabhängiges Organ (Judikative). Selbstverwaltung führt somit nicht nur zu einer Stärkung der demokratischen Legitimation, sondern sie dient auch der Verhinderung von Machtmissbrauch und der Stärkung des Rechtsstaatsprinzips.

\section{DRITTENS}

\section{Exekutive als „Gewährleister" von Justizdienstleistungen versus Verantwortung für eigene Organisationsstrukturen als Ausdruck institutioneller Autonomie}

CONTRA

Exekutive als „Gewährleister“" von Justizdienstleistungen

\section{Verzicht auf Regierungsstimme ist ein Nachteil in der Haushaltsdebatte}

Es werden unterschiedliche Ansichten zu der Frage vertreten, ob die Belange der Justiz besser vertreten werden, wenn die Justiz über ein hierfür zuständiges Regierungsmitglied Sitz und Stimme in der Regierung hat oder wenn die Vertretung ausschließlich eigenen Repräsentanten der Justiz (Selbstverwaltungsorganen) obliegt. Wäre die Justizverwaltung von der Regierungsgewalt autonom, so müssten ihre Repräsentanten die Justizinteressen unmittelbar gegenüber dem Parlament vertreten. Deren Positionen erscheinen jedoch von vornherein geschwächt, weil sie mit dem sonstigen Exekutivapparat nicht vernetzt sind. Die Belange des Justizhaushalts werden nicht ohne Rücksicht auf die allgemeine Haushalts- und Finanzsituation beurteilt werden können, stehen somit immer auch mit den Bewertungen und Vorschlägen zum Gesamthaushalt in Zusammenhang, die die Regierung gegenüber dem Parlament machen muss. Die Justiz aus der Gesamtverantwortung der Regierung herauszubrechen, bedeutet eine Schwächung, da ihre Belange nicht mehr in dieser Gesamtverantwortung überzeugend formuliert, sondern nur noch als korporatives Einzelinteresse dargestellt werden können. Den Repräsentanten der Selbstverwaltungsorgane der Justiz wird zudem der Ruf anhaften, Vertreter von Verbands- bzw. Gruppeninteressen zu sein. Demgegenüber stell die Einbindung der Justizbelange in die Regierungsverantwortung den Zusammenhang mit dem Allgemeinwohl her, dem die Regierung insgesamt verpflichtet ist. Die Aufgabe, Justizbelange im Abwägungsprozess z. B. der Haushaltsgesetzgebung professionell geltend zu machen, lässt sich besser verwirklichen, wenn die Justizverwaltung mit ihrer repräsentativen Spitze in der Regierung selbst ständig vertreten ist und ihr damit aus- 
reichendes politisches Gewicht-auch gegenüber den politischen Kräften im Parlament - zukommt.

2. Sachkonflikte bleiben auch in der Selbstverwaltung die gleichen

Die Stellung der Gerichte in der Verwaltung aller Justizangelegenheiten einschließlich der Personalverwaltung und des Haushaltsvollzugs kann und sollte gestärkt werden. Es ist notwendig, die internen Strukturen in den Gerichtsbarkeiten in den Blick zu nehmen und auf eine angemessene Beteiligung der Vertretungsgremien zu achten.

Eine autonome Justizverwaltung - dies würde auch von ihren Verfechtern nicht bestritten - wäre nicht konfliktfrei, weil sich immer wieder Konflikte zwischen der richterlichen Unabhängigkeit bzw. dem Verständnis hiervon und den Anforderungen der Justizverwaltung ergeben werden. Dies gilt für Personal- wie für Organisationsmaßnahmen. Diese Konflikte sind unabhängig von der Grundsatzfrage, ob die Justizverwaltung in die Regierungsexekutive eingebunden ist oder nicht.

3. Missstände drohen primär durch Machthäufung bei den Obergerichtspräsidenten

Die Kritik an der Justizverwaltung wird häufig zu Unrecht in der Person des Justizministers/der Justizministerin oder in der Institution des Justizministeriums , personifiziert". Stein des Anstoßes sind tatsächlich jedoch oft Entscheidungen oder Abläufe in den Bereichen der Justizverwaltung, die in der Verantwortung der Gerichtsvorstände liegen, denen schon jetzt ein wesentlicher Teil der Justizverwaltungsaufgaben übertragen ist. Bei der Beurteilung der Gründe, mit denen eine autonome Justizverwaltung gefordert wird, sollte danach differenziert werden, welcher Mechanismus, welche Fehlentwicklung o.̈̈. denn tatsächlich ursächlich für den angeführten Missstand ist.

Personalentscheidungen werden in der Regel von den Obergerichtspräsidenten/-innen der jeweiligen Gerichtsbarkeit vorbereitet und letztlich-jedenfalls in Brandenburg - vom Richterwahlausschuss getroffen. Den Obergerichtspräsidenten/-innen kommt in der Personalverwaltung eine - vom Ministerium kaum eingeschränkte-Machtfülle zu. Sie haben-u.a. aufgrund des Beurteilungswesens und der Formulierung von Anforderungsprofilen - eine nahezu unkontrollierte Machtstellung über das Schicksal jeder einzelnen Richterin oder jedes einzelnen Richters, die an feudalistische Strukturen erinnert. Die Beteiligung des Präsidialrates ist umso weniger wirksam, je stärker den Gerichtsvorständen eine faktisch beherrschende Stellung in diesem Gremium zugestanden wird. Es gilt daher, Überlegungen anzustellen, wie die Machtausübung in der Gerichtsbarkeit selbst wirksam kontrolliert und Willkür strukturell verhindert werden kann. Die Isolierung der Justizverwaltung löst dieses Problem nicht, sondern wirft die Justiz nur auf sich selbst zurück. 


\section{PROjektgruppe \\ Konsequenzen institutioneller Autonomie: Selbstorganisation und Selbstkon- trolle}

\section{Haushaltsautonomie als Ausdruck der Selbstorganisation}

Es ist Sache des Gesetzgebers, den verfassungsrechtlich gesicherten Prinzipien einer rechtsstaatlichen Dritten Gewalt Rechnung zu tragen. Erforderlich hierfür sind ausreichende Instanzenzüge und das Erfordernis eines personell gewährleisteten richterlichen Kollegialitätsprinzips als Absicherung justizieller Selbstkontrolle.

Für die Dritte Gewalt muss selbstverständlich dasselbe gelten wie für die beiden anderen Gewalten, nämlich sich in ihren Organisationsbereichen autonom zu verwalten. Das setzt zum einen Haushaltskompetenz und zum anderen Haushaltsbestimmung voraus. Schließlich erfordert es auch eine Rechenschaftspflicht gegenüber den Parlamenten. Verfassungsrechtlich gesicherte Justizgewährung ist dabei - wie bei jeder staatlichen Gewalt - am Prinzip sinnvoller Ressourcenbewirtschaftung auszurichten. Das Bundesverfassungsgericht zeigt den verantwortungsvollen Umgang mit den finanziellen Ressourcen als eigene Angelegenheit und nicht als Vorgabe der Regierung - ohne dass ihm der Vorwurf gemacht wird, lediglich „Vertreter von Verbands- bzw. Gruppeninteressen zu sein“.

Die Verpflichtung auf das Allgemeinwohl trifft nicht nur die Regierung bzw. das den Haushalt beschließende Parlament. Gerade auch die Gerichte garantieren durch die Gewährung von Rechtsschutz und der Herstellung des Rechtsfriedens einen entscheidenden Beitrag zur bürgerlichen Freiheitssicherung. Diese Funktion ist in künftigen Haushaltsberatungen herauszustellen und in den staatlichen Gesamtplan einzufügen. Die Entwicklung von Routinen im Prozess der Haushaltserstellung wird dazu führen, dass kompetente und gewählte Vertreter einer selbstverwalteten Justiz nicht schlechter geeignet sind als politisch berufene Minister. Erfahrungen anderer Länder zeigen, dass das Einbeziehen von Fachkräften aus dem administrativen bzw. Finanzbereich in einen Justizrat von Vorteil sein kann und die durch das Herauslösen aus der Regierung entstehenden Nachteile auszugleichen vermögen.

\section{Selbstverwaltung ist sachgerecht für eine effiziente Ressourcenverwaltung}

Art. 97 GG gewährt den Richterinnen und Richtern Unabhängigkeit in ihrer Rechtsprechung. Diese Gewähr gilt sowohl hinsichtlich des Inhalts der Entscheidung als auch hinsichtlich des Prozesses der Entscheidungsfindung. In Zeiten knapper Haushaltsmittel steigt jedoch auch in der Justiz der Bedarf an ressourcenschonenden Verfahren der Justizgewährung. Eine selbstverwaltete Justiz dürfte die Grenzlinie zwischen unabhängiger Rechtsprechung und effizienter Verwaltungstätigkeit der Gerichte genauer treffen. Das Prinzip sparsamer Haushaltsführung, dem alle Staatsgewalten gleichermaßen unterworfen sind, lässt sich erst dann hinreichend legitimieren und umsetzen, wenn die Justiz mittels eigener Organisationsstrukturen darüber autonom entscheiden kann. 
Dies ergibt sich aus zweierlei Gründen: Zum einen verschärfen sich die Gefährdungen, welche die Übernahme betriebswirtschaftlicher Methoden (sog. Neues Steuerungsmodell) innerhalb der Justiz bergen, gerade durch die Federführung der Exekutive. Die Missbrauchsmöglichkeiten durch Steuerungen mittels Personalhoheit werden minimiert, wenn die Gerichte selbst die Verantwortung für die Erbringung der Justizgewährung innehaben. Zum anderen legen neue betriebswirtschaftliche Verwaltungsmodelle durch ihre Fokussierung auf dezentrale Verwaltungseinheiten und deren Ressourcenautonomie die Einführung von Selbstverwaltungselementen nahe.

\section{Psychologie der Unabhängigkeit erfordert die Befreiung vom Zugriff politischer Steuerungslogik der Exekutive}

\section{- Abschaffung von machtbesetzten Hierarchiestrukturen}

Auch eine autonome Justiz wäre nicht konfliktfrei. Es gilt daher auch darüber nachzudenken, die benannten „feudalistischen Strukturen“ durch Reformen des Beförderungs- und Beurteilungssystems zu vermeiden und so der „kaum eingeschränkten Machtfülle“, die die traditionelle Justizhierarchie ermöglicht, entgegen zu wirken.

\section{- Der psychologische Vorteil: Eine Richterschaft , ohne Hoffnung und Furcht'}

Ungeachtet dessen erwächst aber allein aus einer autonomen Justiz eine für unabhängige Richterinnen und Richter unabdingbare Änderung des Selbstbilds: Wenn die Richterschaft vom Zugriff politischer Steuerungslogik der Exekutive befreit wird, dürfte sie eher in der Lage sein, ein stärkeres Unabhängigkeitsbewusstsein zu entwickeln. Eine derartige Freiheit für die Richterschaft befreit nicht von der alleinigen Bindung an Recht und Gesetz. Im Gegenteil: Ein geschärftes Rollenbewusstsein als unabhängige Dritte Gewalt im Staate dürfte gerade diese Bindung noch verstärken. Das Bundesverfassungsgericht zeigt in der Vielfalt der Persönlichkeiten von Richterinnen und Richtern, dass eine solche Art von Unabhängigkeit die Exekutive häufig überrascht, deren Entscheidungen sie sich aber zumeist unterwirft.

Auch wenn ein empirischer Nachweis im Einzelfall kaum zu erbringen ist, weiß man aus der Organisationssoziologie, dass ein Organisationssystem immer in gewissem Umfang Anpassungsverhalten der Mitglieder bewirkt. Die hierauf gerichtete Erwartungshaltung der Exekutive dürfte sich bei der Auswahl und Ernennung von Richtern, bei Beförderungen und Beurteilungen sowie bei Versetzungen und bei Disziplinierungen niederschlagen, wobei Ausnahmen (auf beiden Seiten) die Regel bestätigen. 
VIERTENS

\section{Gefahr gesellschaftlicher Isolierung versus Legitimationszugewinn für die Justiz durch Vertrauensstärkung}

CONTRA

Es droht gesellschaftliche Isolierung

- Einbindung in das Staatsgefüge verpflichtet Richterschaft zur Anpassung an gesellschaftliche Erwartungen

Eine autonome Justizverwaltung wäre gegenüber allen anderen exekutiven Bereichen isoliert. Formen der Koordination oder Kooperation wären erschwert. Zwar trifft es unzweifelhaft zu, dass gerade die Absicht, Grundsätze der Verwaltungsmodernisierung auf die Justizverwaltung bis hin zu den Arbeitsbedingungen und Arbeitsabläufen der Richterinnen und Richter zu übertragen, zu einem Konflikt mit dem Grundsatz richterlicher Unabhängigkeit führen muss. Organisatorische Maßnahmen, die Auswirkungen auf die Rechtsprechung haben (sollen), können zumindest mittelbar die Eigenständigkeit und Unabhängigkeit der rechtsprechenden Gewalt beeinträchtigen. Dieser Konflikt muss jedoch ausgehalten werden und lässt sich nicht durch eine strukturelle Isolierung der Justiz vermeiden; denn in ihm stellt sich ein Konflikt zwischen richterlichem Selbstverständnis und gesellschaftlichen Erwartungen dar. Die Belange der Rechtsprechung können nicht schlechthin einen unbedingten Vorrang vor anderen (staats-)politischen Zielen beanspruchen, die ihrerseits Verfassungsrang haben.

- Exekutive als notwendiger Vermittler gesellschaftlicher Anforderungen an die Dritte Gewalt

Um den politisch zu verhandelnden Ausgleich der Staatsziele sachgerecht beeinflussen zu können, muss der Bedarf der Justiz substantiiert dargelegt werden können. Hierzu gehört auch, immer wieder die gesellschaftliche Funktion sowie die Eigengesetzlichkeit einer unabhängigen Rechtsprechung zu verdeutlichen. Andererseits verlangt dies die Bereitschaft, rechtspolitische Impulse und gesellschaftliche Erwartungen an die Rechtsprechung aufnehmen zu können. Die Einordnung der Justizverwaltung in die von der Regierungsverantwortung getragene Exekutive unter Leitung eines Justizministeriums, das die Belange der Justiz vertritt und die Unabhängigkeit der Gerichte nach außen verteidigt, erscheint insofern gerade für eine in einem demokratischen Rechtsstaat verankerte Justiz sachgerecht. Eine autonome Justizverwaltung birgt die Tendenz, ein Eigenleben zu entfalten, das sich nicht mehr verständlich machen kann. 
PROjektgruppe

Legitimationszugewinn durch Vertrauensstärkung

\section{- Autonomie ermöglicht Verantwortung und schafft Vertrauen}

Art. 92 des Grundgesetzes lautet: „Die rechtsprechende Gewalt ist den Richtern anvertraut; sie wird (...) durch die Gerichte (...) ausgeübt.“ Dieser Verfassungsauftrag erfordert Neutralität und Unparteilichkeit, die durch eine umfassende Autonomie und eine transparentere Rekrutierung der Richterschaft besser gewährleistet werden können. Damit wird auch die strikte Rechts- und Gesetzesbindung der Richter als Ausfluss des Gewaltenteilungsgrundsatzes bestärkt (Art. 97 Abs. 1 GG). Das alles dürfte die Zustimmung der Bevölkerung zur Justiz und zum Recht erhöhen. Deutlicher lässt sich die Verankerung in der Gesellschaft nicht vermitteln.

\section{- Justizressourcen stehen nicht in Konkurrenz zu staatspolitischen Zielen}

Die Rechtsprechung in Konkurrenz zu „(staats-)politischen Zielen“ zu setzen, erscheint im Hinblick auf die verfassungsrechtlichen Staatsorganisationsprinzipien verfehlt. Als Dritte Gewalt hat sie gerade die Aufgabe, die Verfolgung von Staatszielen nicht mit der Verwirklichung von Grundrechten der Bürgerinnen und Bürger kollidieren zu lassen bzw. diese in ein angemessenes Verhältnis zueinander zu setzen. Folglich kann dieses Argument den Ressourcenanspruch der Justiz nicht schmälern, sondern eher noch steigern.

\section{- Keine exekutive Vermittlung des Volkswillens}

Wenn von unabhängiger Rechtsprechung die Bereitschaft verlangt wird, rechtspolitische Impulse und gesellschaftliche Erwartungen aufzunehmen, ist der Boden der Verfassung verlassen. Im Rahmen einer strikten Rechts- und Gesetzesbindung kann dies nur über die Verabschiedung von Gesetzen im Parlament und die Berücksichtigung von Grundrechten sowie Verfassungsprinzipien bei der Auslegung geschehen.

Soll dem Justizminister diese Funktion der Vermittlung von rechtspolitischen Impulsen und gesellschaftlichen Erwartungen zukommen, erscheint die Dritte Gewalt lediglich als Anhängsel der Exekutive.

\section{- Die Justiz muss ohne politische Rücksichtnahme mit einer Stimme sprechen}

Eine autonome Justiz wird in die Lage versetzt, durch ihre gewählten Repräsentanten Entscheidungen selbstbewusst und unabhängig einer oft unaufgeklärten Öffentlichkeit gegenüber zu legitimieren und zu verdeutlichen. Die Justiz hätte eine Stimme. Politische Rücksichtnahme mit Blick auf Wahlinteressen entfällt. 